\title{
A baseline survey on food safety hazards in commonly consumed food items in Sri-Lanka
}

\author{
L Gamlath*, T Siriwardana and BH Sudasinghe \\ Directorate of Environmental Health, Occupational Health and Food Safety, Ministry of Health, 26, Sri Sangaraja Mawatha, Colombo 10.
}

Submitted: 01 June 2020; Revised: 15 November 2020; Accepted:27 February 2021

\begin{abstract}
In Sri Lanka, a survey was conducted in 2018 to assess the levels of identified hazards in commonly consumed food items. Pesticide residue levels in vegetables, green-leaves and fruits, heavy metals in salt, mercury and formaldehyde in fish, artificial colouring in tea and rice, aflatoxins in chilli powder, peanut and coconut oil and microbiological quality of yoghurt were tested to assess the safety of such foods. 397 fruits, 199 vegetables and 85 green-leave samples were tested for pesticide residues and of them $10.08 \%$ fruit samples, 11.56 $\%$ vegetable samples and $41.18 \%$ green-leave samples were detected with the presence of pesticide residues exceeding Maximum Residue Limits (MRL). Among salt samples tested for lead, arsenic, cadmium and mercury, none of the samples exceeded Maximum Permissible Limits (MPL). About one fifth of red raw rice samples were detected with artificial colouring but none of the tea samples had added artificial colourings. Aflatoxin levels exceeding $10 \mathrm{ppm}$ were detected in $8(10 \%)$ coconut oil, $9(11.25 \%)$ peanut and $15(18.75 \%)$ chilli powder samples. All the yoghurt brands tested complied with microbiological specifications given in SLS standard. This survey provides an overview of the level of contaminants and adulterants in commonly consumed food items in Sri Lanka. It is recommended to carry out regular surveillance to monitor food safety hazards.
\end{abstract}

Keywords: Aflatoxin, artificial colourings, food safety hazards, formaldehyde, heavy metals, pesticide residues.

\section{INTRODUCTION}

A food safety hazard can be defined as a biological, chemical or physical agent in, or condition of, food with the potential to cause an adverse health effect
(Codex Alimentarius Commission, 2018). Food safety hazards are the factors that food safety practice seeks to protect against, contain and eliminate from foods. Food safety has become an important public health concern and many governments have put in place measures to manage the risks arising from food safety hazards. A thorough understanding about the possible presence of these hazards is the first essential step in their control.

Food safety hazards can be introduced to foods during primary production due to unsafe use of inputs and during food processing due to contamination, adulteration and use of permitted food additives exceeding recommended levels. Codex Alimentarius defines a contaminant as 'any biological or chemical agent, foreign matter, or other substances not intentionally added to food which may compromise food safety or suitability' (Codex Alimentarius Commission, 2003). The Codex definition of a contaminant implicitly includes naturally occurring toxicants including toxic metabolites of certain microfungi (mycotoxins) that are not intentionally added to food and feed (Codex Alimentarius Commission, 2015). While contaminants are not intentionally added food safety hazards, food fraud is an economically motivated concept that generally involves intentional actions taken to deceive consumers and compromises the authenticity, safety, quality or reliability of food (Spink, 2019). As a subcategory of food fraud, adulteration of food is the fraudulent substitution or addition of undeclared or unapproved substances to food products for increasing their apparent value or reducing the cost

\footnotetext{
* Corresponding author ((ltgamlath@gmail.com; (iD https://orcid.org/0000-0002-0628-4708)
} 
of their production for economic gain. In agreement with the codex definition of contaminant, an adulterant can be defined as 'any substance intentionally added to food, which is not present in such food as a result of the production' (Spink et al., 2019). Considering intentional added substances, not only unpermitted, but permitted substances (food additives) also pose health risks if not adhere to specified limits for use. Even though greater emphasis is on controlling food safety hazards during food processing, it is equally important to strive in controlling pesticide residues, residues of veterinary drugs, and other feed additives that are the main food safety hazards resulting from primary production due to serious health hazards associated with their high intake into the body.

A public concern exists in Sri Lanka over food safety hazards originating from primary production, contaminants, adulterants and unsafe use of chemicals in commonly consumed food items due to reported and foreseeable health risks. Among others, contaminants such as heavy metals in salt, mercury in fish, aflatoxin in chilli powder, peanut and coconut oil, and microbial quality of food products are given higher attention.

Pesticide residues are a reason for public fear related to food safety arising from primary production of food crops. Over the past decade, there is an increased concern over the use of pesticides worldwide. Pesticide residues in food, as deposits of pesticide active ingredients, their metabolites or breakdown products have potential detrimental effects on human health. In Sri Lanka, authorities are vigilant on pesticide residues in vegetables, fruits and green-leaves and the Department of Agriculture in Sri Lanka has issued Maximum Residue Limits (MRL) for commonly used pesticides in food crops.

Table salt is a commodity frequently contaminated with heavy metals from sea water. The heavy metals of greatest concern in sea water and thus in table salt are, copper, zinc, cadmium, mercury and lead, considering the health risks. Lead adversely affects the haematological, central nervous and renal systems. Cadmium mainly affects the renal functions. Arsenic affects a large number of organs and systems including skin, lung, liver, cardiovascular system, nervous system, haemopoetic system and reproductive system (Hutton, 1987). Since salt is an essential additive, which is routinely added to most foods, not only for improving taste but also as a preservative to many canned, salted and pickled or fresh foods, presence of heavy metals could cause adverse effects among a high percentage of the population.
Similarly, the accumulation of mercury in tissues of certain types of fish has been reported and the preference for fish in Sri Lanka as a protein source poses a possible risk of high mercury intake through fish consumption. Organic mercury compounds are toxic to the central nervous system and teratogenic to the fetus. Mothers exposed to high levels of mercury could give birth to babies with psychomotor retardation, blindness, deafness and seizures (Pandey et al., 2012).

Formaldehyde is a chemical substance and its addition to various foods including fish poses health risks. It is extremely noxious for human health being the cause of a variety of illnesses including different forms of cancer and leukemia. Formaldehyde is identified as a carcinogen in humans by the International Agency for Research on Cancer (IARC) and the National Toxicology Program (World Health Organization, 2012).

Aflatoxins are a group of mycotoxins and high incidence of aflatoxins are reported in food groups including peanuts, chilli, and tree nuts in the field and during storage. Aflatoxin is a potential carcinogen and may adversely affect all organ systems, especially liver and kidneys (Herrman \& Walker, 1999).

A food fraud reported in Sri Lanka is adulteration of used tea for reselling by addition of artificial colourings. Artificial colourants are permitted for use as synthetic colours in specific food items and tea is not included in this list. Colour adulteration is strictly prohibited from a consumer health point of view and colouring of tea has gradually become a matter of serious concern currently.

It is important to assess these hazards in commonly consumed foods to identify the magnitude of the problem and to take remedial measures. Contaminant levels in food can be reasonably reduced through best practices such as Good Agricultural Practice (GAP) and Good Manufacturing Practice (GMP). If good hygiene is not practiced, yoghurt, a dairy product commonly consumed in Sri Lanka, also gets contaminated with spoilage and pathogenic microorganisms. Presence of coliforms usually indicates failures in hygienic practices in food preparation and Escherichia coli indicates faecal contamination leading to possible food safety issues (Macedo et al., 2017). However, microbial quality of yoghurt greatly varies with the brand due to varying degree of best practices followed by the manufacturers.

Maximum levels (MLs)/Maximum Permissible limits (MPL); maximum concentrations of a particular substance recommended to be legally permitted in that 
commodity (Codex Alimentarius Commission, 2019), are specified for these food safety hazards nationally and internationally to ensure the safety of consumers. In Sri Lanka, the competent authority which is responsible to ensure food safety is the Food Control Administration Unit (FCAU) of the Ministry of Health and it is responsible for the protection of consumers' health by ensuring that foods that are imported, produced, marketed, distributed and consumed meet the highest standards of food safety and hygiene by conforming to identified limits. Considering the public concern over food safety and also to ensure supply of safe food by the producers and manufacturers, FCAU took the lead in conducting a baseline survey of these food safety hazards covering a wide range of food contaminants island wide. It is expected to provide evidence based recommendations to producers, manufacturers and consumers to maintain public health standards by ensuring food safety of commonly consumed food items that potentially pose risks to human health.

\section{METHODOLOGY}

\section{Materials and sampling}

Food items and food safety hazards were determined before inception of the survey based on the inputs from experts of the Food Control Administration Unit and Food Advisory Committee. One authorized officer was selected from each Regional Director of Health Services area (RDHS area) for sample collection. RDHA areas are compatible with districts except Ampara District where

Table 1: Pre-determined food items, food safety hazards and planned sample size

\begin{tabular}{|c|c|c|}
\hline Food item & Food safety hazard & Planned sample size \\
\hline $\begin{array}{l}09 \text { types of commonly consumed vegetables, namely, tomato, } \\
\text { brinjal, leeks, carrot, beans, long beans, ridged gourd, } \\
\text { snake gourd, okra (ladies fingers) }\end{array}$ & Pesticide residues & $\begin{array}{l}3 \text { samples each } \\
\times 9 \text { vegetable types } \\
\times 26 \text { RDHS }=702\end{array}$ \\
\hline $\begin{array}{l}2 \text { types of green leaves, namely, gotukola and } \\
\text { mukunuwenna }\end{array}$ & Pesticide residues & $\begin{array}{l}3 \text { samples each } \\
\times 2 \text { green-leave types } \\
\times 26 \text { RDHS }=156\end{array}$ \\
\hline $\begin{array}{l}7 \text { types of commercially grown fruits, namely, papaw, pineapple, } \\
\text { banana, watermelon, mango, guava, local grapes }\end{array}$ & Pesticide residues & $\begin{array}{l}3 \text { samples each } \\
\times 7 \text { types of fruits } \\
\times 26 \text { RDHS }=546\end{array}$ \\
\hline Salt & $\begin{array}{l}\text { Arsenic, Cadmium } \\
\text { Lead, mercury }\end{array}$ & $\begin{array}{l}3 \text { samples each } \times 26 \\
\text { RDHS }=78\end{array}$ \\
\hline Fish samples & Mercury, Formaldehyde & $\begin{array}{l}3 \text { samples each } \times 26 \\
\text { RDHS }=78\end{array}$ \\
\hline Chilli powder & Aflatoxin & $\begin{array}{l}3 \text { samples each } \times 26 \\
\text { RDHS }=78\end{array}$ \\
\hline Peanut & Aflatoxin & $\begin{array}{l}3 \text { samples each } \times 26 \\
\text { RDHS }=78\end{array}$ \\
\hline Coconut oil & Aflatoxin & $\begin{array}{l}3 \text { samples each } \times 26 \\
\text { RDHS }=78\end{array}$ \\
\hline Tea & Artificial colouring & $\begin{array}{l}3 \text { samples each } \times 26 \\
\text { RDHS }=78\end{array}$ \\
\hline Red rice & Artificial colouring & $\begin{array}{l}3 \text { samples each } \times 26 \\
\text { RDHS }=78\end{array}$ \\
\hline Yoghurt & Microbiological & $\begin{array}{l}3 \text { samples each } \times 26 \\
\text { RDHS }=78\end{array}$ \\
\hline
\end{tabular}

RDHS- Regional Director of Health Services area 
it is divided into two RDHS areas, namely, Ampara and Kalmunai. Each selected authorized officer was instructed to collect 3 samples ( $1 \mathrm{~kg}$ each) from each food item listed in Table 1. Three samples representing 3 locations were collected from each item and sampling was done in such a way that the first sample was collected from the biggest market in the centre of the RDHS area and each of the other 2 samples were collected from a marketplace approximately in the middle of the East and middle of the West from the central place. Samples were collected from all 26 RDHS areas from June-December in 2018 and transported to the pre-identified laboratories following standard sample transporting procedures. Solvents, chemicals and reagents used in this study were of HPLC and analytical grades. Subsampling from a composite sample, followed by analytical samples was prepared adhering to sampling principles to support generalizing the results.

\section{Analysis of pesticide residues in selected vegetables, fruits and green-leaves}

Pesticide residues in selected vegetables, greenleaves and fruits were tested at the Government Analyst Department according to the methods given in AOAC 2007.01 (AOAC, 2007). Pesticide residues in foods were tested by acetonitrile extraction and partitioning with magnesium sulfate test method with some modifications. Twenty-one pesticides, namely, bifenthrin, chlorothalonil, chlorpyrifos, diazinon, difenoconazole, dimethoate, dimethomorph, fenobucarb, fenthion, fipronil, flutolanil, isoprothiolane, malathion, metalaxyl, novaluron, oxadixyl, phosalone, profenophos, propamocarb, pyraclostrobin and thiamethoxam were tested.

\section{Analysis of heavy metals in salt}

Salt samples were tested for heavy metals at the laboratory of the Sri Lanka Standard Institute, as per methods described in SLSI standards (SLS 80:2014; SLS 79:2014).

\section{Mercury}

Determination of total mercury content was done according to the standard, which follows cold vapour atomic absorption spectrometric method as described in EuSalt/AS 012-2005 of the SLSI standard.

\section{Arsenic}

Determination of total arsenic content was carried out according to the standard, which follows silver diethyldithiocarbamate photometric method as described in EuSalt/AS 011-2005 of the SLSI standard.

\section{Cadmium}

Cadmium was measured by using flame atomic absorption spectrometric method specified in the standard which is based on EuSalt/AS 014-2005 of the SLSI standard.

\section{Analysis of mercury and formaldehyde in fish}

Mercury and formaldehyde in fish were tested at the National Aquatic Resources Research and Development Agency (NARA) laboratory and reported on wet weight basis.

\section{Mercury}

For the preparation of sample, one gram of homogenized sample was weighed in a microwave digester tube and $10 \mathrm{~mL}$ of concentrated nitric acid was added and kept for $15 \mathrm{~min}$ in a fume hood for pre-digestion. After predigestion, the samples were digested using a microwave accelerated system (CEM-Mars-6). Each sample was analyzed in duplicate. Two blank tubes prepared in a similar manner (but without the muscle) were included in each batch of analysis. Microwave conditions were set at 800 PSI pressure and $200{ }^{\circ} \mathrm{C}$ temperature with a $10 \mathrm{~min}$ holding time.

The digests were allowed to cool to room temperature and the pressure was released carefully by opening the valve. Each digested fish sample was transferred into a $50 \mathrm{~mL}$ volumetric flask and was diluted up to the mark with deionized water. The determination of $\mathrm{Hg}$ was performed using a Cold Vapor Atomic Absorption Spectrometry system (CV-AAS: Varian VGA 77) in a Varian $240 \mathrm{FS}$ atomic absorption spectrophotometer using $\mathrm{SnCl}_{2}$ as a reduction solution throughout the analysis.

\section{Formaldehyde}

Formaldehyde in fish was tested using the AOAC method (Lagace et al., 2003) which was based on steam distillation, followed by spectrophotometry. The adsorption of the formaldehyde/acetylacetone reagent complex was measured at $412 \mathrm{~nm}$.

\section{Analysis of artificial colourings in tea and rice}

Tea and rice samples were tested for artificial colouring compounds by wool dying method followed by paper chromatography at the Food Laboratory of the National Institute of Health Sciences (NIHS), Kalutara. 


\section{Analysis of aflatoxin in chilli powder, peanut and coconut oil}

Chilli powder, peanut and coconut oil samples were tested for aflatoxins using HPLC at the NIHS food laboratory following the SLS method (SLS 962 Part I, 2013) developed from the ISO method (ISO 16050, 2003). The aflatoxins were quantified by reverse-phase HPLC with fluorescence detection and post-column derivatization.

\section{Analysis of microbiology parameters in yoghurt}

Microbiology parameters in yoghurt were tested at the Medical Research Institute as per SLS standards (SLS 516:3, 2013; SLS 516:12, 2013; SLS 516:2:2013).

\section{Coliform and E. coli}

A yoghurt sample (25 g) was weighed aseptically into a sterile conical flask and diluted with Butterfield's phosphate buffer solution (225 mL). After mixing, a decimal dilution series was prepared. Accurately, $1 \mathrm{~mL}$ from each dilution was transferred into three tubes containing lauryl tryptose broth and inverted Durham vials. After 2 days of incubation at $35^{\circ} \mathrm{C}$, gas accumulated Durham tubes containing lauryl tryptose tubes were subjected for confirmatory test.

Coliform: A loopfull of suspension from each gassing lauryl tryptose broth was transferred to a separate brilliant green bile broth tube, inverted with a Durham vial. Tubes were incubated at $35^{\circ} \mathrm{C}$ for 2 days and gassing tubes were recorded. Total coliforms were calculated using a MPN table for three consecutive dilutions.

E. coli: A loopfull of suspension from each gassing lauryl tryptose broth was transferred to a separate $E$. coli medium tube, inverted with a Durham vial. Tubes were incubated at $45{ }^{\circ} \mathrm{C}$ for 2 days and gassing tubes were recorded. E. coli count was calculated using a MPN table for three consecutive dilutions.

\section{Yeasts and Mould}

A yoghurt sample (25 g) was weighed aseptically into a sterile conical flask and diluted with normal saline solution $(225 \mathrm{~mL})$. It was vortexed (1-2 $\mathrm{min})$ and a decimal dilution series was prepared. From each dilution, $1 \mathrm{~mL}$ was transfected into Petri dishes (DM: $10 \mathrm{~cm})$ in triplicate. About $15 \mathrm{~mL}$ of tempered potato dextrose agar medium containing chlortetracycline- $\mathrm{HCl}$ (40 ppm) was added and incubated in dark at $24 \pm 1{ }^{\circ} \mathrm{C}$. After 5 days fungal colonies were enumerated.

\section{Analysis of data}

Data were analysed using SPSS Software. Data were cleaned before analysis. Proportions and percentages of hazards were calculated for each food item in comparison to a recommended standard. For pesticide residues, MRL was identified based on the Control of Pesticides (Time Limits) Regulation published under the Pesticide Act. When national MRL was not specified, Codex Standard was used. When both standards were not available for a particular pesticide, $0.01 \mathrm{mg} / \mathrm{kg}$ was taken as MRL as this is the limit of quantification for pesticide residues at the Government Analyst Department. In addition to MRL, mean, standard deviation (SD), minimum and maximum values were calculated for pesticide residues, aflatoxins, mercury and formaldehyde levels.

\section{RESULTS AND DISCUSSION}

\section{Distribution of test samples}

RDHS areas from where the composite samples of commonly used food items were collected and tested in this survey are depicted in Table 2. Due to practical limitations associated with sample dispatch and storage, it was not possible to analyse some commodities from certain RDHS areas.

\section{Pesticide residues in fruits, vegetables and green- leaves}

In this baseline survey a greater attention was given to identify presence of residues of selected pesticide types to understand the food safety associated with commonly consumed fruits, vegetables and green leaves.

\section{Pesticide residues in fruits}

As depicted in Table 3, residues of selected pesticide types in seven fruit varieties were analysed. The highest percentage of samples with pesticide residues exceeding MRL was detected in guava $(49.18 \%)$ followed by grapes $(28.57 \%)$, but the number of grape samples tested was seven, which was relatively low compared to the number of samples obtained from other fruit types tested. The commonest pesticide detected in guava was Profenofos. Novaluron, Diazinon, Chlorpyrifos, Profenofos, Flutolanil, Bifenthrin, Difenoconazole and Dimethomorph were detected in pineapple and one pineapple sample drawn from Jaffna was positive for six types of pesticides. Novaluron, Diazinon, Flutolanil, Bifenthrin and Difenoconazole were identified in mango, all exceeding MRL. One mango sample collected from Vauniya was positive for all these 5 types of pesticides. 
Table 2: Regional Director of Health Service areas of which the commonly consumed food items were collected and chemical testing was performed

\begin{tabular}{|c|c|c|c|c|c|c|c|c|c|c|c|c|c|c|c|c|c|c|c|c|c|c|c|c|c|}
\hline \multirow[t]{2}{*}{ District } & \multicolumn{25}{|c|}{ Food Items } \\
\hline & 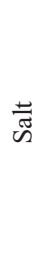 & 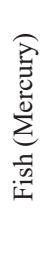 & 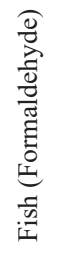 & 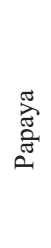 & 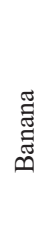 & $\begin{array}{l}8 \\
\stackrel{80}{0} \\
\stackrel{\Xi}{\Sigma}\end{array}$ & 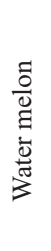 & 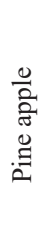 & $\overbrace{0}^{\pi}$ & 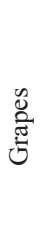 & $\stackrel{\mathscr{E}}{\oplus}$ & $\stackrel{\mathscr{0}}{\approx}$ & 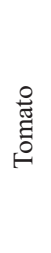 & 营 & $\frac{\tilde{v}}{\stackrel{0}{U}}$ & 节 & 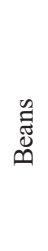 & 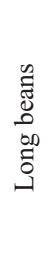 & 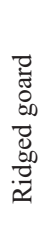 & 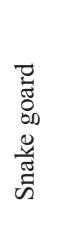 & 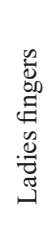 & $\begin{array}{l}\frac{\pi}{0} \\
\frac{0}{3} \\
0 \\
0\end{array}$ & 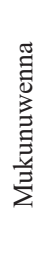 & 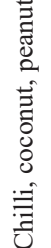 & 䔍 \\
\hline
\end{tabular}

\begin{tabular}{|c|c|c|c|c|c|c|c|c|c|c|c|c|c|c|c|c|c|c|c|c|c|c|c|c|c|}
\hline Colombo & $\sqrt{ }$ & - & - & - & - & - & - & - & - & - & $\sqrt{ }$ & $\sqrt{ }$ & - & - & - & - & - & - & - & - & - & - & - & $\sqrt{ }$ & - \\
\hline Kalutara & $\sqrt{ }$ & - & - & - & - & - & - & - & - & - & $\sqrt{ }$ & $\sqrt{ }$ & - & 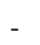 & - & - & - & - & - & - & - & - & - & $\sqrt{ }$ & - \\
\hline Gampaha & $\sqrt{ }$ & - & - & - & - & - & - & - & - & - & $\sqrt{ }$ & $\sqrt{ }$ & - & - & - & - & - & - & - & - & - & - & - & $\sqrt{ }$ & - \\
\hline Kegalle & $\sqrt{ }$ & $\sqrt{ }$ & $\sqrt{ }$ & $\sqrt{ }$ & $\sqrt{ }$ & $\sqrt{ }$ & $\sqrt{ }$ & $\sqrt{ }$ & $\sqrt{ }$ & - & $\sqrt{ }$ & $\sqrt{ }$ & - & 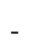 & - & - & 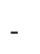 & - & - & - & - & - & - & $\sqrt{ }$ & $\sqrt{ }$ \\
\hline Ratnapura & $\sqrt{ }$ & $\sqrt{ }$ & - & $\sqrt{ }$ & $\sqrt{ }$ & $\sqrt{ }$ & $\sqrt{ }$ & $\sqrt{ }$ & $\sqrt{ }$ & - & $\sqrt{ }$ & $\sqrt{ }$ & $\sqrt{ }$ & $\sqrt{ }$ & $\sqrt{ }$ & $\sqrt{ }$ & - & - & - & - & - & $\sqrt{ }$ & $\sqrt{ }$ & $\sqrt{ }$ & $\sqrt{ }$ \\
\hline Nuwara-Eliya & $\sqrt{ }$ & $\sqrt{ }$ & $\sqrt{ }$ & $\sqrt{ }$ & $\sqrt{ }$ & $\sqrt{ }$ & $\sqrt{ }$ & $\sqrt{ }$ & $\sqrt{ }$ & - & $\sqrt{ }$ & $\sqrt{ }$ & - & - & - & - & - & - & - & - & - & - & - & $\sqrt{ }$ & $\sqrt{ }$ \\
\hline Kandy & $\sqrt{ }$ & - & - & $\sqrt{ }$ & $\sqrt{ }$ & $\sqrt{ }$ & $\sqrt{ }$ & $\sqrt{ }$ & $\sqrt{ }$ & - & $\sqrt{ }$ & $\sqrt{ }$ & - & - & - & - & - & - & - & - & - & - & - & $\sqrt{ }$ & - \\
\hline Matale & $\sqrt{ }$ & $\sqrt{ }$ & $\sqrt{ }$ & $\sqrt{ }$ & $\sqrt{ }$ & $\sqrt{ }$ & $\sqrt{ }$ & $\sqrt{ }$ & $\sqrt{ }$ & - & $\sqrt{ }$ & $\sqrt{ }$ & - & - & - & - & - & - & - & - & - & - & - & $\sqrt{ }$ & $\sqrt{ }$ \\
\hline Jaffna & $\sqrt{ }$ & - & - & $\sqrt{ }$ & $\sqrt{ }$ & $\sqrt{ }$ & - & $\sqrt{ }$ & $\sqrt{ }$ & $\sqrt{ }$ & $\sqrt{ }$ & $\sqrt{ }$ & - & $\sqrt{ }$ & $\sqrt{ }$ & - & 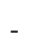 & $\sqrt{ }$ & - & - & - & $\sqrt{ }$ & $\sqrt{ }$ & $\sqrt{ }$ & $\sqrt{ }$ \\
\hline Kilinochchi & $\sqrt{ }$ & - & - & - & $\sqrt{ }$ & $\sqrt{ }$ & $\sqrt{ }$ & $\sqrt{ }$ & $\sqrt{ }$ & $\sqrt{ }$ & $\sqrt{ }$ & $\sqrt{ }$ & - & $\sqrt{ }$ & - & $\sqrt{ }$ & - & $\sqrt{ }$ & . & $\sqrt{ }$ & - & - & - & $\sqrt{ }$ & $\sqrt{ }$ \\
\hline Vavuniya & $\sqrt{ }$ & - & - & $\sqrt{ }$ & $\sqrt{ }$ & $\sqrt{ }$ & - & $\sqrt{ }$ & $\sqrt{ }$ & - & $\sqrt{ }$ & $\sqrt{ }$ & $\sqrt{ }$ & $\sqrt{ }$ & - & - & - & - & - & $\sqrt{ }$ & - & $\sqrt{ }$ & $\sqrt{ }$ & $\sqrt{ }$ & $\sqrt{ }$ \\
\hline Mullaitivu & $\sqrt{ }$ & - & - & $\sqrt{ }$ & $\sqrt{ }$ & $\sqrt{ }$ & $\sqrt{ }$ & $\sqrt{ }$ & $\sqrt{ }$ & $\sqrt{ }$ & $\sqrt{ }$ & $\sqrt{ }$ & - & $\sqrt{ }$ & $\sqrt{ }$ & $\sqrt{ }$ & - & $\sqrt{ }$ & - & $\sqrt{ }$ & - & - & - & $\sqrt{ }$ & $\sqrt{ }$ \\
\hline Mannar & $\sqrt{ }$ & - & - & $\sqrt{ }$ & $\sqrt{ }$ & $\sqrt{ }$ & $\sqrt{ }$ & $\sqrt{ }$ & $\sqrt{ }$ & - & $\sqrt{ }$ & $\sqrt{ }$ & - & $\sqrt{ }$ & $\sqrt{ }$ & - & - & - & 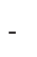 & - & - & $\sqrt{ }$ & $\sqrt{ }$ & $\sqrt{ }$ & $\sqrt{ }$ \\
\hline Kalmunai & $\sqrt{ }$ & $\sqrt{ }$ & $\sqrt{ }$ & $\sqrt{ }$ & $\sqrt{ }$ & $\sqrt{ }$ & $\sqrt{ }$ & $\sqrt{ }$ & $\sqrt{ }$ & - & $\sqrt{ }$ & $\sqrt{ }$ & $\sqrt{ }$ & $\sqrt{ }$ & - & $\sqrt{ }$ & - & - & . & - & - & $\sqrt{ }$ & $\sqrt{ }$ & $\sqrt{ }$ & $\sqrt{ }$ \\
\hline Batticaloa & $\sqrt{ }$ & $\sqrt{ }$ & $\sqrt{ }$ & $\sqrt{ }$ & $\sqrt{ }$ & $\sqrt{ }$ & $\sqrt{ }$ & $\sqrt{ }$ & $\sqrt{ }$ & - & $\sqrt{ }$ & $\sqrt{ }$ & $\sqrt{ }$ & $\sqrt{ }$ & $\sqrt{ }$ & $\sqrt{ }$ & - & - & . & - & - & $\sqrt{ }$ & $\sqrt{ }$ & $\sqrt{ }$ & $\sqrt{ }$ \\
\hline Trincomalee & $\sqrt{ }$ & - & - & $\sqrt{ }$ & $\sqrt{ }$ & $\sqrt{ }$ & $\sqrt{ }$ & $\sqrt{ }$ & $\sqrt{ }$ & - & $\sqrt{ }$ & $\sqrt{ }$ & $\sqrt{ }$ & $\sqrt{ }$ & - & - & - & - & - & $\sqrt{ }$ & $\sqrt{ }$ & $\sqrt{ }$ & - & $\sqrt{ }$ & $\sqrt{ }$ \\
\hline Ampara & $\sqrt{ }$ & $\sqrt{ }$ & $\sqrt{ }$ & $\sqrt{ }$ & $\sqrt{ }$ & $\sqrt{ }$ & $\sqrt{ }$ & $\sqrt{ }$ & $\sqrt{ }$ & - & $\sqrt{ }$ & $\sqrt{ }$ & $\sqrt{ }$ & $\sqrt{ }$ & $\sqrt{ }$ & $\sqrt{ }$ & $\sqrt{ }$ & - & - & - & $\sqrt{ }$ & $\sqrt{ }$ & - & $\sqrt{ }$ & $\sqrt{ }$ \\
\hline Badulla & $\sqrt{ }$ & $\sqrt{ }$ & $\sqrt{ }$ & $\sqrt{ }$ & $\sqrt{ }$ & $\sqrt{ }$ & $\sqrt{ }$ & $\sqrt{ }$ & $\sqrt{ }$ & - & $\sqrt{ }$ & $\sqrt{ }$ & $\sqrt{ }$ & $\sqrt{ }$ & $\sqrt{ }$ & $\sqrt{ }$ & - & - & - & - & - & $\sqrt{ }$ & $\sqrt{ }$ & $\sqrt{ }$ & $\sqrt{ }$ \\
\hline Monaragala & $\sqrt{ }$ & $\sqrt{ }$ & $\sqrt{ }$ & $\sqrt{ }$ & $\sqrt{ }$ & $\sqrt{ }$ & $\sqrt{ }$ & $\sqrt{ }$ & 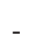 & - & $\sqrt{ }$ & $\sqrt{ }$ & $\sqrt{ }$ & $\sqrt{ }$ & $\sqrt{ }$ & $\sqrt{ }$ & - & - & - & - & - & $\sqrt{ }$ & $\sqrt{ }$ & $\sqrt{ }$ & $\sqrt{ }$ \\
\hline Puttalam & $\sqrt{ }$ & - & - & $\sqrt{ }$ & $\sqrt{ }$ & $\sqrt{ }$ & $\sqrt{ }$ & $\sqrt{ }$ & $\sqrt{ }$ & $\sqrt{ }$ & $\sqrt{ }$ & $\sqrt{ }$ & - & - & - & - & - & - & - & - & - & - & - & $\sqrt{ }$ & - \\
\hline Kurunegala & $\sqrt{ }$ & - & - & $\sqrt{ }$ & $\sqrt{ }$ & $\sqrt{ }$ & $\sqrt{ }$ & $\sqrt{ }$ & $\sqrt{ }$ & - & $\sqrt{ }$ & $\sqrt{ }$ & - & - & - & - & - & - & - & - & - & - & - & $\sqrt{ }$ & $\sqrt{ }$ \\
\hline Anuradhapura & $\sqrt{ }$ & - & - & $\sqrt{ }$ & $\sqrt{ }$ & $\sqrt{ }$ & - & $\sqrt{ }$ & $\sqrt{ }$ & - & $\sqrt{ }$ & $\sqrt{ }$ & $\sqrt{ }$ & $\sqrt{ }$ & - & - & $\sqrt{ }$ & $\sqrt{ }$ & $\sqrt{ }$ & $\sqrt{ }$ & $\sqrt{ }$ & $\sqrt{ }$ & $\sqrt{ }$ & $\sqrt{ }$ & $\sqrt{ }$ \\
\hline Polonnaruwa & - & - & - & $\sqrt{ }$ & $\sqrt{ }$ & $\sqrt{ }$ & - & $\sqrt{ }$ & $\sqrt{ }$ & - & $\sqrt{ }$ & $\sqrt{ }$ & - & - & - & - & - & - & - & - & - & - & - & $\sqrt{ }$ & - \\
\hline Galle & $\sqrt{ }$ & $\sqrt{ }$ & $\sqrt{ }$ & $\sqrt{ }$ & $\sqrt{ }$ & $\sqrt{ }$ & $\sqrt{ }$ & $\sqrt{ }$ & $\sqrt{ }$ & - & $\sqrt{ }$ & $\sqrt{ }$ & $\sqrt{ }$ & $\sqrt{ }$ & - & - & $\sqrt{ }$ & $\sqrt{ }$ & $\sqrt{ }$ & $\sqrt{ }$ & $\sqrt{ }$ & $\sqrt{ }$ & $\sqrt{ }$ & $\sqrt{ }$ & $\sqrt{ }$ \\
\hline Matara & $\sqrt{ }$ & $\sqrt{ }$ & $\sqrt{ }$ & $\sqrt{ }$ & $\sqrt{ }$ & $\sqrt{ }$ & $\sqrt{ }$ & $\sqrt{ }$ & $\sqrt{ }$ & $\sqrt{ }$ & $\sqrt{ }$ & $\sqrt{ }$ & $\sqrt{ }$ & $\sqrt{ }$ & - & - & $\sqrt{ }$ & $\sqrt{ }$ & $\sqrt{ }$ & $\sqrt{ }$ & $\sqrt{ }$ & $\sqrt{ }$ & $\sqrt{ }$ & $\sqrt{ }$ & $\sqrt{ }$ \\
\hline Hambanthota & $\sqrt{ }$ & $\sqrt{ }$ & $\sqrt{ }$ & $\sqrt{ }$ & $\sqrt{ }$ & $\sqrt{ }$ & $\sqrt{ }$ & $\sqrt{ }$ & $\sqrt{ }$ & - & $\sqrt{ }$ & $\sqrt{ }$ & $\sqrt{ }$ & $\sqrt{ }$ & $\sqrt{ }$ & $\sqrt{ }$ & - & $\sqrt{ }$ & $\sqrt{ }$ & $\sqrt{ }$ & - & $\sqrt{ }$ & $\sqrt{ }$ & $\sqrt{ }$ & $\sqrt{ }$ \\
\hline
\end{tabular}

$\sqrt{ }$ - tested; - not tested 
Table 3: $\quad$ Pesticide residues in fruits

\begin{tabular}{|c|c|c|c|c|c|c|c|c|c|}
\hline Food item & No. (\%) of & No. (\%) & & Des & tion of pesti & ides detect & & & \\
\hline $\begin{array}{l}\text { and no.a } \\
\text { of samples } \\
\text { tested (n) }\end{array}$ & $\begin{array}{c}\text { samples } \\
\text { detected with } \\
\text { pesticides }^{\mathrm{a}}\end{array}$ & $\begin{array}{c}\text { of samples } \\
\text { above } \\
\text { MRL }^{\mathrm{a}}\end{array}$ & Name & $\begin{array}{l}\mathrm{MRL} \\
\mathrm{mg} / \mathrm{kg}\end{array}$ & $\begin{array}{c}\text { No. }(\%) \\
\text { above } \\
\text { MRL }\end{array}$ & $\begin{array}{c}\text { No. }(\%) \\
\text { below } \\
\text { MRL }\end{array}$ & $\begin{array}{c}\text { Mean } \\
(\mathrm{SD}) \\
(\mathrm{mg} / \mathrm{kg})^{\mathrm{e}}\end{array}$ & $\begin{array}{l}\text { Minimum- } \\
\text { maximum } \\
(\mathrm{mg} / \mathrm{kg})^{\mathrm{f}}\end{array}$ & $\begin{array}{c}\text { RDHS areas } \\
\text { above MRL }\end{array}$ \\
\hline $\begin{array}{l}\text { Papaya } \\
(\mathrm{n}=65)\end{array}$ & Papaya & $01(1.54)$ & Fenobucarb & 0.01 & - & $1(1.54)$ & 0.01 & - & \\
\hline & & & Oxadixyl & 0.01 & $1(1.54)$ & - & 0.28 & - & Batticoloa \\
\hline $\begin{array}{l}\text { Banana } \\
(\mathrm{n}=71)\end{array}$ & $01(1.41)$ & $00(0.0)$ & Difenoconazole & $0.1^{\mathrm{c}}$ & - & $1(1.41)$ & 0.08 & - & \\
\hline $\begin{array}{l}\text { Watermelon } \\
(\mathrm{n}=60)\end{array}$ & $01(1.67)$ & $01(1.67)$ & Oxadixyl & 0.01 & $1(1.67)$ & - & 0.06 & - & Ampara \\
\hline $\begin{array}{l}\text { Pineapple } \\
(\mathrm{n}=69)\end{array}$ & $06(8.7)$ & $03(4.35)$ & Bifenthrin & 0.01 & - & $1(1.45)$ & 0.01 & - & \\
\hline & & & $\begin{array}{l}\text { Chlorpyrifos } \\
\text { Diazinon }\end{array}$ & $\begin{array}{l}0.01 \\
0.1^{\mathrm{c}}\end{array}$ & $\begin{array}{c}1(1.45) \\
-\end{array}$ & $1(1.45)$ & $\begin{array}{l}0.02 \\
0.01\end{array}$ & $\begin{array}{l}- \\
-\end{array}$ & Jaffna \\
\hline & & & Difenoconazole & 0.01 & $1(1.45)$ & - & 0.05 & - & Jaffna \\
\hline & & & Dimethomorph & $0.01^{\mathrm{c}}$ & $1(1.45)$ & - & 0.03 & - & Jaffna \\
\hline & & & Flutolanil & 0.01 & - & $1(1.45)$ & 0.01 & - & \\
\hline & & & Novaluron & 0.01 & $1(1.45)$ & - & 0.02 & - & Mannar \\
\hline & & & Profenophos & $0.05^{\mathrm{d}}$ & $1(1.45)$ & $3(4.35)$ & $\begin{array}{c}0.04 \\
(0.03)\end{array}$ & $0.02-0.08$ & Kandy \\
\hline Guava & $37(60.66)$ & $30(49.18)$ & Bifenthrin & 0.01 & - & $1(1.64)$ & 0.01 & - & \\
\hline (Kilopera) & & & Difenoconazole & 0.01 & - & $2(3.28)$ & 0.01 & - & \\
\hline$(\mathrm{n}=61)$ & & & Dimethomorph & 0.01 & $1(1.64)$ & - & 0.22 & - & Jaffna \\
\hline & & & Profenophos & 0.01 & $29(47.54)$ & $6(9.84)$ & $\begin{array}{l}0.054 \\
(0.05)\end{array}$ & $0.01-0.24$ & Names $^{\mathrm{b}}$ \\
\hline Grapes & $03(42.86)$ & $2(28.57)$ & Difenoconazole & $3.0^{\mathrm{c}}$ & - & $1(14.29)$ & 0.03 & - & \\
\hline$(n=7)$ & & & Profenophos & 0.01 & $2(28.57)$ & - & $\begin{array}{c}0.04 \\
(0.01)\end{array}$ & $0.03-0.05$ & $\begin{array}{c}\text { Jaffna } \\
\text { Kilinochchi }\end{array}$ \\
\hline \multirow[t]{5}{*}{$\begin{array}{l}\text { Mango } \\
(n=64)\end{array}$} & $02(3.13)$ & $02(3.13)$ & Bifenthrin & 0.01 & $2(3.13)$ & - & $\begin{array}{l}0.075 \\
(0.08)\end{array}$ & $0.02-0.13$ & $\begin{array}{c}\text { Vavuniya } \\
\text { Batticoloa }\end{array}$ \\
\hline & & & Diazinon & 0.01 & $1(1.56)$ & - & 0.02 & - & Vavuniya \\
\hline & & & Difenoconazole & $0.07^{\mathrm{c}}$ & $1(1.56)$ & - & 0.09 & - & Vavuniya \\
\hline & & & Flutolanil & 0.01 & $1(1.56)$ & - & 0.02 & - & Vavuniya \\
\hline & & & Novaluron & 0.01 & $1(1.56)$ & - & 0.08 & - & Vavuniya \\
\hline $\begin{array}{l}\text { Total } \\
(n=397)\end{array}$ & $52(13.1)$ & $40(10.08)$ & & & & & & & \\
\hline
\end{tabular}

a: Some samples were positive for several pesticides

b: Kilinochchi, Polonnaruwa, Hambanthota, Mannar, Mullaitivu, Puttalum, Kurunegala, Matara, Galle, Ratnapura, Badulla, Trincomalee,

Ampara, Kalmunai, Batticoloa, Jaffna, Vavuniya

c: codex value; d: Sri Lankan value

e: When one particular pesticide type was detected in a single sample per each food item, SD is not applicable

f: When one particular pesticide type was detected in a single sample per each food item, minimum and maximum value are not applicable 
Table 4: Pesticide residues in vegetables

\begin{tabular}{|c|c|c|c|c|c|c|c|c|c|}
\hline \multirow{2}{*}{$\begin{array}{l}\text { Food item and } \\
\text { no. of samples } \\
\text { tested (n) }\end{array}$} & \multirow{2}{*}{$\begin{array}{l}\text { No. }(\%) \text { of } \\
\text { samples } \\
\text { detected with } \\
\text { pesticides }^{a}\end{array}$} & \multirow{2}{*}{$\begin{array}{l}\text { No. }(\%) \text { of } \\
\text { samples } \\
\text { above } \\
\text { MRL }^{a}\end{array}$} & \multicolumn{7}{|c|}{ Description of pesticides detected } \\
\hline & & & Name & $\begin{array}{l}\mathrm{MRL} \\
\mathrm{mg} / \mathrm{kg}\end{array}$ & $\begin{array}{l}\text { No. }(\%) \\
\text { above MRL }\end{array}$ & $\begin{array}{l}\text { No. }(\%) \\
\text { below } \\
\text { MRL }\end{array}$ & $\begin{array}{l}\text { mean } \\
(\mathrm{SD}) \\
(\mathrm{mg} / \\
\mathrm{kg})^{\mathrm{e}}\end{array}$ & $\begin{array}{l}\text { Minimum- } \\
\text { maximum } \\
(\mathrm{mg} / \mathrm{kg})^{\mathrm{f}}\end{array}$ & $\begin{array}{l}\text { RDHS areas } \\
\text { above MRL }\end{array}$ \\
\hline \multirow[t]{3}{*}{$\begin{array}{l}\text { Tomato } \\
(\mathrm{n}=34)\end{array}$} & $14(41.18)$ & $07(20.59)$ & Fenobucarb & 0.01 & $5(14.71)$ & $4(11.76)$ & $\begin{array}{l}0.017 \\
(0.01)\end{array}$ & $0.01-0.03$ & $\begin{array}{l}\text { Trincomalee } \\
\text { Badulla } \\
\text { Moneragala }\end{array}$ \\
\hline & & & Novaluron & $0.02^{\mathrm{d}}$ & $2(5.88)$ & $2(5.88)$ & $\begin{array}{l}0.06 \\
(0.05)\end{array}$ & $0.01-0.12$ & $\begin{array}{l}\text { Batticoloa } \\
\text { Kalmunai }\end{array}$ \\
\hline & & & Profenophos & $10.0^{\mathrm{c}}$ & - & $4(11.76)$ & $\begin{array}{l}0.06 \\
(0.04)\end{array}$ & $0.02-0.12$ & \\
\hline \multirow{4}{*}{$\begin{array}{l}\text { Brinjal } \\
(n=44)\end{array}$} & $07(15.91)$ & $04(9.09)$ & Diazinon & 0.01 & $1(2.27)$ & - & 0.17 & - & Kalmunai \\
\hline & & & Fenobucarb & 0.01 & - & $2(4.55)$ & $\begin{array}{l}0.01 \\
(0.00)\end{array}$ & $0.01-0.01$ & \\
\hline & & & Flutolanil & 0.01 & $2(4.55)$ & $2(4.55)$ & $\begin{array}{l}0.04 \\
(0.04)\end{array}$ & $0.01-0.07$ & $\begin{array}{l}\text { Batticoloa } \\
\text { Trincomalee }\end{array}$ \\
\hline & & & Profenophos & 0.01 & $1(2.27)$ & - & 0.02 & - & Ampara \\
\hline \multirow[t]{2}{*}{$\begin{array}{l}\text { Leeks } \\
(\mathrm{n}=23)\end{array}$} & $02(8.7)$ & $01(4.35)$ & Diazinon & 0.01 & $1(4.35)$ & - & 0.02 & - & Batticoloa \\
\hline & & & Pyraclostrobin & $0.7^{\mathrm{d}}$ & - & $1(4.35)$ & 0.01 & - & \\
\hline \multirow[t]{2}{*}{$\begin{array}{l}\text { Carrot } \\
(n=23)\end{array}$} & $03(13.04)$ & 03 (13.04) & Dimethomorph & 0.01 & $1(4.35)$ & - & 0.03 & - & Hambanthota \\
\hline & & & Profenophos & 0.01 & $2(8.7)$ & - & $\begin{array}{l}0.62 \\
(0.54)\end{array}$ & $0.23-1.0$ & $\begin{array}{l}\text { Badulla } \\
\text { Moneragala }\end{array}$ \\
\hline $\begin{array}{l}\text { Beans } \\
(\mathrm{n}=8)\end{array}$ & $01(12.5)$ & $01(12.5)$ & Profenophos & 0.01 & $1(12.5)$ & - & 0.02 & - & Galle \\
\hline $\begin{array}{l}\text { Long beans } \\
(\mathrm{n}=20)\end{array}$ & $03(15.0)$ & $03(15.0)$ & Profenophos & 0.01 & $3(15)$ & - & $\begin{array}{l}0.15 \\
(0.2)\end{array}$ & $0.03-0.38$ & $\begin{array}{l}\text { Matara } \\
\text { Galle } \\
\text { Jaffna }\end{array}$ \\
\hline \multirow[t]{2}{*}{$\begin{array}{l}\text { Ridged gourd } \\
(\mathrm{n}=11)\end{array}$} & $02(18.18)$ & $00(0)$ & Metalaxyl & 0.01 & - & $1(9.09)$ & 0.01 & - & \\
\hline & & & Profenophos & $0.05^{\mathrm{d}}$ & - & $1(9.09)$ & 0.03 & - & \\
\hline \multirow[t]{2}{*}{$\begin{array}{l}\text { Snakegourd } \\
(\mathrm{n}=23)\end{array}$} & 05 (21.74) & 04 (17.39) & Diazinon & 0.01 & $1(4.35)$ & - & 0.04 & - & Trincomalee \\
\hline & & & Profenophos & $0.05^{\mathrm{d}}$ & $3(13.04)$ & $1(4.35)$ & $\begin{array}{l}0.17 \\
(0.15)\end{array}$ & $0.02-0.32$ & $\begin{array}{l}\text { Hambanthot } \\
\text { Mullaitivu } \\
\text { Anuradhapu }\end{array}$ \\
\hline $\begin{array}{l}\text { Okra } \\
(\mathrm{n}=13)\end{array}$ & $00(0)$ & $00(0)$ & - & - & - & - & - & - & \\
\hline $\begin{array}{l}\text { Total } \\
(\mathrm{n}=199)\end{array}$ & $37(18.59)$ & $23(11.56)$ & & & & & & & \\
\hline
\end{tabular}

a: Some samples were positive for several pesticides

c: codex value; d: Sri Lankan value

e: When one particular pesticide type was detected in a single sample per each food item, SD is not applicable

f: When one particular pesticide type was detected in a single sample per each food item, minimum and maximum value are not applicable

Guava was the most commonly contaminated fruit found in this survey and is one of the commonly available fruits in Sri Lanka.
As depicted in Table 4, nine types of vegetables were tested for pesticide residues. Of the vegetables tested, percentage of samples detected with the presence of 
Table 5: Pesticide residues in green-leaves

\begin{tabular}{|c|c|c|c|c|c|c|c|c|c|}
\hline Food item and & No. $(\%)$ of & No. & & & Descript & on of pesti & des detected & & \\
\hline $\begin{array}{l}\text { no. of samples } \\
\text { tested (n) }\end{array}$ & $\begin{array}{c}\text { samples } \\
\text { detected } \\
\text { with } \\
\text { pesticides }^{a}\end{array}$ & $\begin{array}{c}(\%) \text { of } \\
\text { samples } \\
\text { above } \\
\text { MRL }^{a}\end{array}$ & Name & $\begin{array}{l}\mathrm{MRL} \\
\mathrm{mg} / \mathrm{kg}\end{array}$ & $\begin{array}{l}\text { No. }(\%) \\
\text { above MRL }\end{array}$ & $\begin{array}{l}\text { No. }(\%) \\
\text { below } \\
\text { MRL }\end{array}$ & $\begin{array}{l}\text { mean }(\mathrm{SD}) \\
(\mathrm{mg} / \mathrm{kg})^{\mathrm{e}}\end{array}$ & $\begin{array}{l}\text { Minimum- } \\
\text { maximum } \\
(\mathrm{mg} / \mathrm{kg})^{\mathrm{f}}\end{array}$ & $\begin{array}{l}\text { RDHS areas } \\
\text { above MRL }\end{array}$ \\
\hline \multirow[t]{6}{*}{$\begin{array}{l}\text { Mukunuwenna } \\
(\mathrm{n}=44)\end{array}$} & $22(50.00)$ & $\begin{array}{c}18 \\
(40.91)\end{array}$ & Chlorothalonil & 0.01 & $8(18.18)$ & $1(2.27)$ & $3.39(6.78)$ & $0.01-19.37$ & $\begin{array}{l}\text { Batticoloa } \\
\text { Jaffna } \\
\text { Badulla } \\
\text { Moneragala }\end{array}$ \\
\hline & & & Flutolanil & 0.01 & $2(4.55)$ & $1(2.27)$ & $0.10(0.08)$ & $0.01-0.16$ & $\begin{array}{l}\text { Vavuniya } \\
\text { Ratnapura }\end{array}$ \\
\hline & & & Metalaxyl & 0.01 & $4(9.09)$ & - & $\begin{array}{l}0.11 \\
(0.054)\end{array}$ & $0.06-0.17$ & $\begin{array}{l}\text { Mullaitivu } \\
\text { Vavuniya } \\
\text { Moneragala }\end{array}$ \\
\hline & & & Novaluron & 0.01 & $3(6.82)$ & - & $1.2(0.22)$ & $0.95-1.36$ & Jaffna \\
\hline & & & Profenophos & 0.01 & $5(11.36)$ & $2(4.55)$ & $0.09(0.09)$ & $0.01-0.22$ & $\begin{array}{l}\text { Hambanthota } \\
\text { Matara } \\
\text { Mannar } \\
\text { Anuradhapura }\end{array}$ \\
\hline & & & Pyraclostrobin & 0.01 & $2(4.55)$ & - & $0.3(0.4)$ & $0.02-0.58$ & Moneragala \\
\hline \multirow[t]{6}{*}{$\begin{array}{l}\text { Gotukola } \\
(\mathrm{n}=41)\end{array}$} & $19(46.34)$ & $\begin{array}{c}17 \\
(41.46)\end{array}$ & Chlorothalonil & 0.01 & $1(2.44)$ & - & 0.02 & - & Ampara \\
\hline & & & Diazinon & 0.01 & $1(2.44)$ & $2(4.88)$ & $0.23(0.02)$ & $0.01-0.05$ & Trincomalee \\
\hline & & & Fenthion & 0.01 & $1(2.44)$ & - & 0.02 & - & Ratnapura \\
\hline & & & Metalaxyl & 0.01 & $2(4.88)$ & - & $0.06(0.02)$ & $0.04-0.08$ & Ratnapura \\
\hline & & & Profenophos & 0.01 & $13(31.71)$ & $1(2.44)$ & $2.79(5.96)$ & $0.01-21.98$ & $\begin{array}{l}\text { Ampara } \\
\text { Hambanthota } \\
\text { Galle } \\
\text { Trincomalee } \\
\text { Jaffna } \\
\text { Badulla } \\
\text { Moneragala } \\
\text { Anuradhapura }\end{array}$ \\
\hline & & & Pyraclostrobin & 0.01 & $1(2.44)$ & & 0.22 & - & Ratnapura \\
\hline Total $(n=85)$ & $41(48.24)$ & $\begin{array}{c}35 \\
(41.18)\end{array}$ & & & & & & & \\
\hline
\end{tabular}

a: Some samples were positive for several pesticides

c: codex value; d: Sri Lankan value

e: When one particular pesticide type was detected in a single sample per each food item, SD is not applicable

$\mathrm{f}$ : When one particular pesticide type was detected in a single sample per each food item, minimum and maximum values are not applicable

at least one type of pesticide residues were $41.8 \%$ in tomato, $21.74 \%$ in snake gourd, $18.18 \%$ in ridged gourd, $15.91 \%$ in brinjal and $15.0 \%$ in long beans. The highest percentage of samples with pesticide residues exceeding MRL was detected in tomato (20.59\%). Pesticides were not identified in okra. The commonest pesticide detected in vegetable samples was Profenofos. Pesticide residues identified in tomato were Novaluron, Fenobucarb and Profenofos while the commonest was Profenofos. However, the detected amount of Profenofos was well 
below the MRL. Profenofos was the only pesticide detected in beans and long beans, and the detected levels exceeded MRL.

In this survey, green leafy vegetables were considered separately from other vegetables because consumers are highly concerned about the possible availability of pesticide residues in green leaves consumed in the country. As depicted in Table 5, two fifth of 'mukunuwenna' and 'gotukola' samples were detected with pesticide residues above MRL.

This survey assessed 397 fruits, 199 vegetables, and 85 green leaf samples for pesticide residues and of them $13.1 \%$ of fruit samples, $18.59 \%$ of vegetable samples and $48.24 \%$ of green leaf samples were detected with the presence of at least one type of pesticide. Pesticide residues exceeding MRL were detected in $10.08 \%$ fruit samples, $11.56 \%$ vegetable samples and $41.18 \%$ greenleaf samples. Dimethoate, Fipronil, Isoprothiolane, Malathion, Phosalone, Propamocarb and Thiamethoxam were not detected in any of the food items tested for pesticide residues.

According to the results, it can be interpreted that the risk of pesticide residues is highest in green-leaves, followed by vegetables, and it is lower in fruits studied in this survey. However, facilities were available to check only 21 pesticide types and all the samples were not tested for these 21 pesticides. Some samples were detected with the presence of several pesticide types and all the three samples collected from some RDHS areas were positive for pesticides. A higher risk is posed by samples having residues of several pesticides especially when they exceeded the MRL. It is noticeable that guava is posing a higher risk to consumers compared to other fruit types. Next to guava, grapes also pose a considerable risk that consumers should be aware of. In terms of vegetables, tomato exerts the highest risk compared to other vegetable types.

A study conducted by Lakshani et al. (2017) to evaluate the presence of pesticides in selected vegetables sold in the open markets in Nuwara-Eliya, Puttlam and Matale districts have found pesticide residues in vegetables including tomato, capsicum and cabbage. Of 90 samples tested, 30 (33.3\%) were contaminated with pesticide residues, which is higher than in the present survey. Similar to the present survey, they emphasize the higher percentage of pesticide residues in tomato. Based on the findings of this survey, they recommend that monitoring studies should be expanded to other districts. Further in Sri Lanka, an issue exists with regard to the use of unpermitted pesticide types. A group of researchers in Sri Lanka has conducted a similar study (Padmajani et al., 2014) to estimate the level of pesticides used and to investigate the factors affecting overuse or misuse. In this study potato, beans, cabbage and leeks were collected from selected areas in the Badulla and Nuwara-Eliya districts. According to their findings, about $5 \%$ of the active ingredients of pesticides used in upcountry vegetable farming belong to unpermitted Class (ib) type, while another $34 \%$ belong to the category of restricted use [Class (ii)]. About $47 \%$ of the farmers prefer to use organophosphate (OP) group of insecticides, as they believe these pesticides give quick results and are cheaper despite their toxicity and harmful nature on the environment.

\section{Heavy metals in salt}

As depicted in Table 6, 84 salt samples were tested for lead, arsenic, cadmium and mercury. None of the samples were detected with levels above MPL. Levels detected for lead, arsenic, cadmium, and mercury were less than $1.0 \mathrm{mg} / \mathrm{kg}, 0.1 \mathrm{mg} / \mathrm{kg}, 0.2 \mathrm{mg} / \mathrm{kg}$ and $0.1 \mathrm{mg} /$ $\mathrm{kg}$, respectively.

Although several countries report the presence of heavy metals in table salt, based on this survey, heavy metals such as arsenic, lead, cadmium and mercury levels in table salts sold in markets in Sri Lanka do not exceed MPL, conforming to national standards. Furthermore, there are no reported studies in Sri Lanka which indicate the presence of heavy metals in table salts sold in markets. This may be associated with good practices related to production, to meet specifications in standards.

Table 6: Heavy metals in salt

\begin{tabular}{lccc}
\hline Parameter & $\begin{array}{c}\text { MPL } \\
(\mathrm{mg} / \mathrm{Kg})\end{array}$ & $\begin{array}{c}\text { No. of samples } \\
\text { tested }\end{array}$ & $\begin{array}{c}\text { No. (\%) below } \\
\text { MPL }\end{array}$ \\
\hline Lead $(\mathrm{Pb})$ & 2.0 & 84 & $84(100 \%)$ \\
Arsenic $(\mathrm{As})$ & 0.5 & 84 & $84(100 \%)$ \\
Cadmium $(\mathrm{Cd})$ & 0.5 & 84 & $84(100 \%)$ \\
Mercury $(\mathrm{Hg})$ & 0.1 & 84 & $84(100 \%)$ \\
\hline
\end{tabular}


Table 7: Mercury and formaldehyde in fish

\begin{tabular}{lcccccc}
\hline Parameter & $\begin{array}{c}\text { No. of samples } \\
\text { tested }\end{array}$ & $\begin{array}{c}\text { MPL } \\
(\mathrm{mg} / \mathrm{kg})\end{array}$ & $\begin{array}{c}\text { No. (\%) detected } \\
\text { but below MPL }\end{array}$ & $\begin{array}{c}\text { No. (\%) detected } \\
\text { with above MPL }\end{array}$ & $\begin{array}{c}\text { Mean (SD) } \\
(\mathrm{mg} / \mathrm{kg})\end{array}$ & $\begin{array}{c}\text { Minimum and maximum } \\
(\mathrm{mg} / \mathrm{kg})\end{array}$ \\
\hline Mercury & 71 & $0.5^{\mathrm{c}}$ & $31(43.66)$ & $00(0)$ & $0.18(0.09)$ & $0.07-0.42$ \\
Formaldehyde & 72 & $5.0^{\mathrm{d}}$ & $10(13.89)$ & $02(2.78)$ & $2.09(2.46)$ & $0.18-7.16$ \\
\hline
\end{tabular}

\section{Mercury and formaldehyde in fish}

As depicted in Table 7, mercury and formaldehyde content were tested in around seventy samples of fish. Mercury was detected in 31 (43.66\%) samples out of 71 fish samples tested, but all were below MPL. Twelve $(16.67 \%)$ fish samples were identified as having formaldehyde but only two $(2.78 \%$ ) fish samples drawn from Kegalle $(6.81 \mathrm{mg} / \mathrm{kg}$ ) and Moneragala $(7.16 \mathrm{mg} / \mathrm{kg})$ districts exceeded MPL.

The mercury levels in fish samples studied in this survey were below the MPL. In contrast, Jinadasa et al. (2010) have reported that, $57 \%$ of swordfish samples exceeded the MPL of European legislation $(1 \mathrm{mg} / \mathrm{kg})$ while none of the samples from yellowfin tuna and snapper exceeded the MPL. The research has recommended that higher levels of mercury in swordfish need to be considered, when dealing with particularly sensitive population groups such as pregnant woman, lactating mothers, infants and children. Jinadasa et al. (2014) again have reported that they have studied mercury levels in swordfish, yellowfin tuna, black marlin and red snapper. Among these fish types swordfish ( 0.90 $\mathrm{mg} / \mathrm{kg}$, wet weight) showed the highest, and the lowest was recorded for red snapper $(0.16 \mathrm{mg} / \mathrm{kg}$, wet weight $)$. Mean total mercury concentration of yellowfin tuna and black marlin was 0.30 and $0.49 \mathrm{mg} / \mathrm{kg}$ wet weight, respectively. However, total mercury in yellowfin tuna and red snapper has not exceeded the MPL while marlin and swordfish exceeded the MPL. The same study has observed that total mercury levels vary in different months of the year, and in swordfish higher levels were observed in August and September. Mean mercury levels of the present survey are even below the levels detected by Jinadasa et al. $(2010,2014)$.

In Sri Lanka, regulations on formaldehyde in fish came into operation in 2010 prohibiting the import, transport, distribution, storage, sale, offering or exposure for sale any fish in Sri Lanka, which contains formaldehyde at levels exceeding $5 \mathrm{mg} / \mathrm{kg}$ (ppm). In this survey, about $17 \%$ of fish samples showed the presence of formaldehyde and about $3 \%$ exceeded the MPL. A study conducted by Madusha et al. (2017) have assessed formaldehyde levels in sword fish, squids, Indian scad and bullet tuna species and reported that the values are in the range of $0.917-3.34 \mathrm{mg} / \mathrm{kg}$, well below the MPL. However, attention needs to be given to assure that market-sold fish do not exceed safe limits.

\section{Artificial colouring in raw red rice and tea}

About one fifth of the red raw rice samples were detected with artificial colouring and these samples were drawn from Colombo, Ampara, Badulla and Mullaitivu RDHS areas. The commonest colouring used was sunset yellow (E 110). Both Tartrazine (E 102) and sunset yellow were identified in one red rice sample drawn from Galle RDHS area. Both these colourings are permitted in Sri Lanka, but not allowed to be used in rice or tea.

Tea is the most commonly consumed beverage in Sri Lanka and none of the analysed samples showed any artificial colouring. With the understanding received from these data, surveillance programmes are currently in progress to identify possible adulteration of rice and tea with artificial colourants.

\section{Aflatoxins in coconut oil, peanut and chilli powder}

As depicted in Table 9, aflatoxin was tested in eighty samples each of coconut oil, peanut and chilli powder. Aflatoxin levels exceeding $10 \mathrm{ppm}$ were detected in 8 $(10 \%)$ coconut oil, $9(11.25 \%)$ peanut and 15 (18.7 $5 \%)$ chilli powder samples. More than one tenth of chilli powder samples were detected with aflatoxins exceeding MRL (30 ppm). These samples were drawn from Colombo, Kalutara, Nuwara-Eliya, Baticoloa, Matale, Gampaha and Ampara RDHS areas. Peanut samples drawn from Mullaitivu, Kilinochchi, Baticoloa and Kegalle RDHS areas showed aflatoxin levels above $30 \mathrm{ppm}$ and three peanut samples were detected with values above $100 \mathrm{ppm}$, with the highest being 417.86 
ppm (Baticoloa: 120.71 ppm, Kilinochchi: 200.57 ppm and Mullaitivu: $417.86 \mathrm{ppm}$ ). Aflatoxin level more than $30 \mathrm{ppm}$ was detected only in one coconut oil sample drawn from Trincomalee RDHS area.

SLS standard for coconut oil has specified the MPL of total aflatoxin level in coconut oil as $10 \mathrm{ppm}$. Codex Alimentarius also has specified the MPL for aflatoxin as $10 \mathrm{ppm}$ for many products. According to Sri Lankan regulations in operation, MPL for total aflatoxin is 30 ppm for the food items mentioned in Table 9. Another study conducted in Sri Lanka to assess aflatoxin levels in edible vegetable oil types have also revealed that out of 32 coconut oil samples 12 (37.5\%), which included 5 branded and 7 unbranded products, had total aflatoxin contamination and aflatoxin $\mathrm{B}_{1}$ contamination in the ranges of $2.25-72.70 \mu \mathrm{g} / \mathrm{kg}$ and $1.76-60.92 \mu \mathrm{g} / \mathrm{kg}$, respectively. Of these 12 samples, 10 and 11 samples exceeded the European Union's maximum permissible levels applicable for total aflatoxins $(4 \mu \mathrm{g} / \mathrm{kg})$ and aflatoxin $\mathrm{B}_{1}(2 \mu \mathrm{g} / \mathrm{kg})$, respectively (Karunarathna et al., 2019). However, the present survey did not focus on analyzing aflatoxin $\mathrm{B}_{1}$ levels separately.

Table 8: Artificial colouring in raw red rice and tea

\begin{tabular}{lcc}
\hline Food item & Total No. of samples & No. $(\%)$ detected \\
\hline Red raw rice & 82 & $16(19.51 \%)$ \\
Tea & 81 & $0(0 \%)$ \\
\hline
\end{tabular}

Table 9: Aflatoxins in coconut oil, peanut and chilli powder

\begin{tabular}{lcccccc}
\hline Food item & $\begin{array}{c}\text { Total No. } \\
\text { of samples }\end{array}$ & $\begin{array}{c}\text { Detected }<10 \\
\text { ppm No. }(\%)\end{array}$ & $\begin{array}{c}\geq 10 \mathrm{ppm} \\
\text { No. }(\%)\end{array}$ & $\begin{array}{c}\geq 30 \mathrm{ppm} \\
\text { No. }(\%)\end{array}$ & $\begin{array}{c}\text { Mean (SD) } \\
(\mathrm{ppm})\end{array}$ & $\begin{array}{c}\text { Minimum - } \\
\text { maximum (ppm) }\end{array}$ \\
\hline Coconut Oil & 80 & $29(36.25)$ & $8(10)$ & $1(1.25)$ & $9.04(1.2)$ & $1.86-68.71$ \\
Peanut & 80 & $28(35.00)$ & $9(11.25)$ & $4(5.00)$ & $26.05(7.6)$ & $1.86-417.86$ \\
Chilli Powder & 80 & $27(33.75)$ & $15(18.75)$ & $9(11.25)$ & $18.45(2.79)$ & $1.86-152.29$ \\
\hline
\end{tabular}

\section{Microbiology parameters in yoghurt}

Forty two locally available yoghurt samples were tested each for presumptive coliform count, Escherichia coli count, yeast count, mould count, and all the samples complied with microbiological specifications given in SLS standards for yoghurt. Strict hygienic practices and protocols followed by Sri Lankan dairy factories may be the reason for this. In contrast, Hemamali et al. (2016) have conducted a study to assess physico-chemical and microbiological parameters of some plain set yoghurt sold in Matara municipal area of Sri Lanka. Five different brands of plain set yoghurt samples were collected on the basis of consumer preference and have reported that all microbiological parameters of all yoghurt brands were not within the permissible range of local and international standards. In line with their findings, Rathnayaka and Silva (2013) have reported that total plate count and total yeast and mould count of some commercial set yoghurt samples were not within the SLS standards. Therefore, continuous surveillance would be the strategy to monitor food safety and quality parameters of yoghurt brands available in the market.

This survey provides an overview of the level of contamination and adulteration in commonly consumed food items in Sri Lanka. However, there were a few limitations in terms of testing samples of some commodities from all RDHS areas due to practical difficulties.

Table 10: Bacterial contaminations in yoghurt

\begin{tabular}{lcc}
\hline $\begin{array}{l}\text { Bacteriological } \\
\text { parameter }\end{array}$ & $\begin{array}{c}\text { Total no. of } \\
\text { samples }\end{array}$ & No. (\%) satisfactory \\
\hline Coliform count & 42 & $42(100)$ \\
Escherichia coli count & 42 & $42(100)$ \\
Yeast count & 42 & $42(100)$ \\
Mould count & 42 & $42(100)$ \\
\hline
\end{tabular}




\section{CONCLUSION AND RECOMMENDATIONS}

Pesticide residues exceeding MRL were detected in $10.08 \%$ fruit samples, $11.56 \%$ vegetable samples and $41.18 \%$ green-leaf samples. Salt was free from heavy metals. Mercury and formaldehyde were detected in fish samples but mercury levels did not exceed the MPL. Artificial colouring was not detected in tea but was in red rice. Aflatoxin levels exceeding $30 \mathrm{ppm}$ were detected in $1.25 \%$ coconut oil, $5 \%$ peanut and $11.25 \%$ chilli powder samples. Yoghurt samples were free from microbiological contamination. A multisectoral approach is needed to further reduce the level of contaminants in food. It is recommended that awareness campaigns to be carried out throughout the country to educate farmers, distributers and sellers on adverse effects of contaminated food, and farmers instructed to follow GAP in farming. Surveillance programmes to monitor the contaminants and adulterants in food items is also recommended.

\section{Acknowledgements}

Authors thank the authorized officers of Ministry of Health for the support provided in sample collection, the staff of food laboratory of National Institute of Health Science, Kalutara; Government Analyst Department; Sri Lanka Standard Institute; National Aquatic Resources Research and Development Agency (NARA) and microbiology laboratory of Medical Research Institute, Borella for conducting relevant analysis. The authors also acknowledge the support provided by the officers attached to the Directorate of Environmental Health, Occupational Health and Food Safety in surveillance programmes. This survey was funded by the Ministry of Health, Sri Lanka.

\section{REFERENCES}

Association of Official Analytical Chemists (AOAC) (2007). AOAC Official Method 2007.01. Pesticide Residues in Foods by Acetonitrile Extraction and Partitioning with Magnesium Sulfate. Official Methods of Analysis of AOAC International, USA.

Codex Alimentarius Commission (2003). Codex Codes of Practice for General Principles of Food Hygiene: CAC/ RCP 1-1969, WHO-FAO, Rome, Italy.

Codex Alimentarius Commission (2015). Codex General Standard for Contaminants and Toxins in Food and Feed, CODEX STAN 193-1995, WHO-FAO, Rome, Italy.

Codex Alimentarius Commission (2018). Procedural Manual, Twenty Sixth Edition, WHO-FAO, Rome, Italy.
Codex Alimentarius Commission (2019). Codex General Standard for Food Additives, CODEX STAN 192-1995, Rev. 3-2001, WHO-FAO, Rome, Italy.

Hutton M. (1987). Human health concerns of lead, mercury, cadmium and arsenic. In: Lead, Mercury, Cadmium and Arsenic in the Environment (eds. T.C. Hutchinson \& K.M. Meema). pp. 53-68. John Wiley \& Sons Ltd, New Jersey, USA.

Hemamali K.K.G.U., Jayamanne V.S. \& Amarathunge S.M. (2016). Physico-chemical and microbiological quality of some consumer preferred plain set yoghurts sold in Matara municipal area of Sri Lanka. International Journal of Scientific \& Technology Research 5(01): 62-65.

Herrman J.L. \& Walker R. (1999). Risk Analysis of Mycotoxins, Joint FAO/WHO Expert Committee on Food Additives (JECFA). FNA/ANA 23: 17-24.

Jinadasa B.K.K.K., Rameesha L.R.S., Edirisinghe E.M.R.K.B. \& Rathnayake R.M.U.S.K. (2010). Mercury, cadmium and lead levels in three commercially important marine fish species in Sri Lanka. Sri Lanka Journal of Aquatic Science 15: $39-43$.

DOI: https://doi.org/10.4038/sljas.v15i0.5481

Jinadasa, B.K.K.K., Edirisinghe E.M.R.K.B. \& Wickramasinghe I. (2014). Total mercury, cadmium and lead levels in main export fish of Sri Lanka. Food Additives and Contaminants: Part B: Surveillance 7(4): 309-314. DOI: https://doi.org/10.1080/19393210.2014.938131

Karunarathna N.B., Fernando C.J., Munasinghe D.M.S. \& Fernando R. (2019). Occurrence of aflatoxins in edible vegetable oils in Sri Lanka. Food Control 101: 97-103.

DOI: https://doi.org/10.1016/j.foodcont.2019.02.017

Lagace L., Guay S. \& Martin N. (2003). Level of endogenous formaldehyde in maple syrup as determined by spectroscopy. Journal of AOAC International 86: 598-601. DOI: https://doi.org/10.1093/jaoac/86.3.598

Lakshani P.W.Y., Rajapaksha M.K.L.K. \& Sendthuran K. (2017). Pesticide residues in selected vegetables in several growing areas by GC/MS using QuEChERS Technique. Annals of Sri Lanka Department of Agriculture 19(2): 188-208.

DOI: https://doi.org/10.4038/ouslj.v13i1.7427

Macedo K.M., da Silva I.M.M., de Oliveira F.S., Castro J.T., dos Santos D.C.M.B., Freitasb F. \& de Jesusa M.C. (2017). Toxic elements and microbiological content of food: Evidence from a case study in a Brazilian city heavily contaminated by lead and cadmium. Journal of the Brazilian Chemical Society 28(7): 1220-1228. DOI: http://doi.org/10.21577/0103-5053.20160283

Madusha P.G.D., Ganegamaarachchi G.J., Ginigaddarage P.H., Ariyawansa K.W.S., Wickramasinghe I., Hettiarachchi K.S., Abhayarathne S. \& Perera R. (2017). Quality assessment of imported fish retailed in Sri Lanka. Proceedings of the National Aquatic Resources Research and Development 
Agency (NARA), Scientific Sessions. p. 61.

Padmajani M.T., Aheeyar M.M.M. \& Bandara M.M.M. (2014). Assessment of Pesticide Usage in Up-Country Vegetable Farming in Sri Lanka, HARTI Research Report No: 164, Hector Kobbekaduwa Agrarian Research and Training Institute, Colombo.

Pandey G., Madhuri S. \& Shrivastav A.B. (2012). Contamination of mercury in fish and its toxicity to both fish and humans: an overview. International Research Journal of Pharmacy 3(11): 44-47.

Rathnayaka R.M.U.S.K. \& De Silva K.L.S.R. (2013). Physicochemical sensory and microbiological evaluation of set and fruit yoghurt in Sabaragamuwa Province, Sri Lanka. Journal of Scientific Research and Reports 3(2): 284-293.

DOI: https://doi.org/10.9734/JSRR/2014/6512

Spink J. (2019). The current state of food fraud prevention: Overview and requirements to address "How to Start?" and "How Much is Enough?" Current Opinions in Food Science 27: 130-138.

DOI: https://doi.org/10.1016/j.cofs.2019.06.001

Spink J., Bedard B., Keogh J., Moyer D.C., Scimeca J. \& Vasan A. (2019). International survey of food fraud and related terminology: preliminary results and discussion. Journal of Food Science 84(10): 2705-2718.

DOI: https://doi.org/10.1111/1750-3841.14705

Sri Lanka Standards Institution (SLSI) (2013). SLS 516 Part 2 Section 1, Standard for Methods of test for Microbiology of Food and Animal Feeding Stuffs - Horizontal Method for the Enumeration of Yeasts and Moulds - Colony count technique in products with water activity greater than 0.95. Sri Lanka Standards Institution, Colombo.

Sri Lanka Standards Institution (SLSI) (2013). SLS 516 Part 3 Section 1, Standard for Methods of Test for Microbiology of Food and Animal Feeding Stuffs - Horizontal Method for the Detection and Enumeration of Coliforms - Most Probable Number Technique. Sri Lanka Standards Institution, Colombo.

Sri Lanka Standards Institution (SLSI) (2013). SLS 516 Part 12, Methods of test for Microbiology of Food and Animal Feeding Stuffs - Horizontal Method for the Detection and Enumeration of Presumptive Escherichia coli (Most Probable Number Technique). Sri Lanka Standards Institution, Colombo.

Sri Lanka Standards Institution (SLSI) (2013). SLS 962 Part I, Standard for Method of Test for Aflatoxin in Foods Determination of Aflatoxin B1, and the Total Content of Aflatoxins B1, B2, G1, and G2 in Cereals, Nuts and Derived Products - High-performance Liquid Chromatographic Method. Sri Lanka Standards Institution, Colombo.

Sri Lanka Standards Institution (SLSI) (2014). SLS 79, Edible Iodized/ Non-iodized Salt (Granular form). Sri Lanka Standards Institution, Colombo.

SriLanka Standards Institution(SLSI) (2014). SLS 80 (Powdered form) Standard for Edible Iodized salt (Powdered form). Sri Lanka Standards Institution, Colombo.

World Health Organization (2012). Chemical Agents and Related Occupations. IARC Monographs on the Evaluation of Carcinogenic Risks to Humans, volume 100F. The International Agency for Research on Cancer, Lyon, France. 